TRANSACTIONS OF THE

AMERICAN MATHEMATICAL SOCIETY

Volume 363, Number 4, April 2011, Pages 1687-1698

S 0002-9947(2010)05483-2

Article electronically published on November 2, 2010

\title{
ALL THE GIT QUOTIENTS AT ONCE
}

\author{
NICHOLAS PROUDFOOT
}

\begin{abstract}
Let $G$ be an algebraic torus acting on a smooth variety $V$. We study the relationship between the various GIT quotients of $V$ and the symplectic quotient of the cotangent bundle of $V$.
\end{abstract}

Let $G$ be a reductive algebraic group acting on a smooth variety $V$. The cotangent bundle $T^{*} V$ admits a canonical algebraic symplectic structure, and the induced action of $G$ on $T^{*} V$ is Hamiltonian, that is, it admits a natural moment map $\mu: T^{*} V \rightarrow \mathfrak{g}^{*}$ (see Equation (1) for an explicit formula). Over the past ten years, a guiding principle has emerged that says that if $X$ is an interesting variety which may be naturally presented as a GIT (geometric invariant theory) quotient of $V$ by $G$, then the symplectic quotient $\mu^{-1}(\lambda) / / G$ of $T^{*} V$ by $G$ is also interesting. This mantra has been particularly fruitful on the level of cohomology, as we describe below. Over the complex numbers, a GIT quotient may often be interpreted as a Kähler quotient by the compact form of $G$, and an algebraic quotient as a hyperkähler quotient. For this reason, the symplectic quotient may be loosely thought of as a quaternionic or hyperkähler analogue of $X$. Let us review a few examples of this construction.

Hypertoric varieties. These examples comprise the case where $G$ is abelian and $V$ is a linear representation of $G$. The geometry of toric varieties is deeply related to the combinatorics of polytopes; for example, Stanley $\mathrm{St}$ ] used the hard Lefschetz theorem for toric varieties to prove certain inequalities for the $h$-numbers of a simplicial polytope. The hyperkähler analogues of toric varieties, known as hypertoric varieties, interact in a similar way with the combinatorics of rational hyperplane arrangements. Introduced by Bielawski and Dancer BD, hypertoric varieties were used by Hausel and Sturmfels [HS] to give a geometric interpretation of virtually every known property of the $h$-numbers of a rationally representable matroid. Webster and the author $\mathrm{PW}$ extended this line of research by studying the intersection cohomology groups of singular hypertoric varieties.

Quiver varieties. A quiver is a directed graph, and a representation of a quiver is a vector space for each node along with a linear map for each edge. For any quiver, Nakajima [N1, N2, N3] defined a quiver variety to be the quaternionic analogue of the moduli space of framed representations. Examples include the Hilbert scheme of $n$ points in the plane and the moduli space of instantons on an ALE space. He has shown that the cohomology and K-theory groups of quiver varieties carry actions of

Received by the editors February 17, 2006 and, in revised form, March 23, 2006.

2010 Mathematics Subject Classification. Primary 14L24; Secondary 14L30, 53D20.

This research was partially supported by a National Science Foundation Postdoctoral Research Fellowship.

(C) 2010 American Mathematical Society Reverts to public domain 28 years from publication 1687 
Kac-Moody algebras and their associated quantum algebras, and has exploited this fact to define canonical bases for highest weight representations. Crawley-Boevey and Van den Bergh [CBVdB] and Hausel [Ha] have used Betti numbers of quiver varieties to prove a long standing conjecture of Kac.

Hyperpolygon spaces. Given an ordered $n$-tuple of positive real numbers, the associated polygon space is the moduli space of $n$-sided polygons in $\mathbb{R}^{3}$ with edges of the prescribed lengths, up to rotation. Such a space may be interpreted as a moduli space of stable configurations of points on a projective line, or, via the Gelfand-MacPherson correspondence [HK], as a GIT quotient of the Grassmannian $G(2, n)$ by the natural action of the torus $T^{n-1}$. The quaternionic analogues of these spaces were introduced by Konno $\underline{\mathrm{K} 2}$, and dubbed hyperpolygon spaces in $\mathrm{HP}$. In $[\mathrm{HP}$, Harada and the author show that certain projective subvarieties of a hyperpolygon space have interpretations in terms of spacial polygons, which suggests the general problem of searching for moduli-theoretic interpretations of hyperkähler analogues of moduli spaces. While hyperpolygon spaces are in fact special cases of quiver varieties, they will be of special interest to us in this paper because they may be constructed using an abelian group.

To define a GIT quotient of a variety $V$ by a group $G$ one needs more data than just an action; to be precise, we need a $G$-equivariant ample line bundle on $V$. If we define two such line bundles to be equivalent whenever they lead to the same GIT quotient, there will in general be finitely many distinct equivalent classes of equivariant ample line bundles. In the toric case, these classes correspond to triangulations of an oriented matroid [Sa]. In the case of polygon spaces, the choice of line bundle corresponds to the choice of edge lengths. The various GIT quotients of $V$ by $G$ will always be birational, but will generally be topologically distinct.

The symplectic quotient of $T^{*} V$ by $G$ requires two choices, namely an equivariant line bundle as well as an element $\lambda \in\left(\mathfrak{g}^{*}\right)^{G}$ at which to reduce. If $G$ is abelian and $\lambda$ is chosen generically, however, then $G$ will act locally freely on $\mu^{-1}(\lambda)$, and the symplectic quotient

$$
M_{\lambda}:=\mu^{-1}(\lambda) / / G=\mu^{-1}(\lambda) / G
$$

will not depend on the choice of line bundle. In fact, the topological type of the symplectic quotient over the complex numbers does not depend on the choice of generic $\lambda$, either! Intuitively, this can be seen from the fact that the set of generic parameters $\lambda$ is connected, but the noncompactness of the quotients makes this argument technically difficult. In this paper we take a different approach, proving the following theorem (Corollary 1.4).

Theorem. If $G$ is abelian and $\lambda \in \mathfrak{g}^{*}$ is a regular value for $\mu$, then $M_{\lambda}$ is isomorphic to the total space of an affine bundle over the nonseparated prevariety obtained by gluing together the various smooth GIT quotients of $V$ along the open sets on which they agree.

This surprising result, taken over the complex numbers, implies that the topology of any one symplectic quotient of $T^{*} V$ is intimately related to the topology of all of the different GIT quotients of $V$. It is for this reason that we use the phrase 'all the GIT quotients at once'. We note that the theorem in fact holds over arbitrary fields, and was used in $[\mathrm{PW}$ to count points on hypertoric varieties over finite fields. 
Section 1 is devoted to giving a careful definition of the various objects referred to in the above theorem. The proof itself is remarkably simple, following CrawleyBoevey's work on quiver varieties in [CB]. In Section 2 we consider the natural map from the cohomology ring of $M_{\lambda}$ to the direct sum of the cohomology rings of the GIT quotients, whose existence follows immediately from the theorem. Konno K2, 7.6] proves that this map is an injection in the case of hyperpolygon spaces 1 , and we prove the analogous theorem for hypertoric varieties (Theorem 2.8). We conjecture that such a result will hold in greater generality (Conjecture 2.1). A consequence of this conjecture would be that the cohomology ring of $M_{\lambda}$ is level, meaning that it satisfies an analogue of Poincaré duality for noncompact spaces (see Remark 2.4). In the case of hypertoric varieties, this is a well-known and nontrivial fact [HS, §7], but it is by no means the case for every smooth manifold.

If $G$ is allowed to be nonabelian, the above theorem will fail. To fix it we would have to replace the base by a stack which contains the union of the GIT quotients as an open subscheme, and interpret the affine bundle in a suitable stacky context (see Remark 1.5). One can formulate an analogue of Conjecture 2.1 in the nonabelian case, but we do not feel that enough evidence exists for such a conjecture at the present time.

\section{An AFFine BUNDLE}

Let $V$ be a smooth algebraic variety over an arbitrary field $k$. We will assume that $V$ is projective over affine, which means that the natural map $V \rightarrow \operatorname{Spec} \mathcal{O}_{V}$ is projective. Let $G$ be an algebraic torus over $k$ acting effectively on $V$, and let $L$ be a $G$-equivariant ample line bundle on $V$. A point $p \in V$ is called $L$-semistable if there exists a $G$-invariant section of a positive power of $L$ that does not vanish at $p$. The set of $L$-semistable points of $V$ will be denoted $V^{s s}(L)$. An $L$-semistable point $p$ is called $L$-stable if $G$ acts locally freely at $p$ and its orbit is closed in $V^{s s}(L)$. The set of $L$-stable points of $V$ will be denoted $V^{s t}(L)$. If every $L$-semistable point is $L$-stable, then we will call $L$ nice.

We will consider two equivariant ample line bundles to be equivalent if they induce the same stable and semistable sets. Let $\left\{L_{i} \mid i \in I\right\}$ be a complete set of representatives of equivalence classes of nice line bundles with nonempty stable sets. Let $V^{\ell f}$ denote the set of points of $V$ at which $G$ acts locally freely. By definition, $V^{s t}(L)$ is contained in $V^{\ell f}$ for any $L$. The following lemma is a converse to this fact.

Lemma 1.1. Suppose that there exists at least one ample equivariant line bundle on $V$. If $G$ acts locally freely at $p$, then $p$ is L-stable for some nice $L$, thus we have $V^{\ell f}=\bigcup_{i \in I} V^{s t}\left(L_{i}\right)$.

Proof. For any point $p \in V$, and any equivariant line bundle $L$, choosing an identification of $L_{p}$ with $k$ gives us a natural element $\operatorname{ev}(p) \in \Gamma(L)^{\vee}$, the dual of the vector space of sections of $L$. The equivariant structure on $L$ gives a decomposition

$$
\Gamma(L)^{\vee}=\bigoplus_{\chi} \Gamma(L)_{\chi}^{\vee}
$$

\footnotetext{
${ }^{1}$ Konno does not phrase his theorem in these terms, as he does not have Corollary 1.4 at his disposal. But it is easy to translate his result into the one that we attribute to him.
} 
where $\chi$ ranges over the lattice $\operatorname{Char}(G)=\operatorname{Hom}\left(G, \mathbb{G}_{m}\right)$. Let ev $(p)_{\chi}$ be the component of $\operatorname{ev}(p)$ corresponding to the character $\chi$. The state polyhedron $\Delta_{p}(L) \subseteq$ $\operatorname{Char}(G)_{\mathbb{Q}}$ is defined to be the convex hull inside of $\operatorname{Char}(G)_{\mathbb{Q}}$ of the set $\{\chi \mid$ $\left.\mathrm{ev}(p)_{\chi} \neq 0\right\}$. Dolgachev and $\mathrm{Hu}[\mathrm{DH}, 1.1 .5]$ show that $p$ is $L$-semistable if and only if the trivial character is contained in $\Delta_{p}(L)$, and $L$-stable if and only if it is contained in the interior of $\Delta_{p}(L)$. The affine span of $\Delta_{p}(L)$ is equal to a shift of the set of rational characters that vanish on the stabilizer of $p$, where the shift is given by the character with which the stabilizer of $p$ acts on $L_{p}$. In particular, the interior of $\Delta_{p}(L)$ is nonempty if and only if $G$ acts locally freely at $p$.

Let $L_{\chi}$ be the trivial bundle on $V$ with equivariant structure given by the character $\chi$. Then

$$
\Delta_{p}\left(L^{\otimes m} \otimes L_{\chi}\right)=m \cdot \Delta_{p}(L)-\chi,
$$

thus by taking a high enough tensor power of $L$ and twisting it by an appropriate character, we may find a new equivariant ample line bundle whose state polytope is an arbitrary dilation and translation of that of $L$. In particular, if $p \in V^{\ell f}$, we may find an $L$ with respect to which $p$ is stable. It remains to show that this line bundle can be chosen to be nice. For all $q \notin V^{\ell f}, \Delta_{q}(L)$ is contained in a proper affine subspace of $\operatorname{Char}(G)_{\mathbb{Q}}$. Let $\chi$ be a character which is nontrivial on the stabilizer of $q$ for every $q \in V \backslash V^{\ell f}$. By again replacing $L$ with a large tensor power and twisting by $\chi$, we may ensure that none of these subspaces contains the origin. If $\chi$ is chosen to be small with respect to the size of the tensor power, then this operation will not break the $L$-stability of $p$.

For any ample equivariant line bundle $L$, the GIT (geometric invariant theory) quotient

$$
V /{ }_{L} G:=V^{s s}(L) / G
$$

is defined to be the categorical quotient of $V^{s s}(L)$ by $G$, in which two points are identified if the closures of their $G$-orbits intersect. The fact that $V$ is projective over $\operatorname{Spec} \mathcal{O}_{V}$ implies that $V /{ }_{L} G$ is projective over $\operatorname{Spec} \mathcal{O}_{V}^{G}$. If $L$ is nice, then $V /{ }_{L} G$ is simply the geometric quotient of $V^{s t}(L)$ by $G$. For all $i \in I$, let $X_{i}=V / /_{L_{i}} G$ be the corresponding GIT quotient.

Example 1.2. Let $V=G(2, n)$ be the Grassmannian of 2-planes in $\mathbb{C}^{n}$, and let $G=T^{n} / \mathbb{C}_{\text {diag }}^{\times}$be the $(n-1)$-torus acting on $V$. The GIT quotients $\left\{X_{i}\right\}$ can also be realized as GIT quotients of an $n$-fold product of projective lines by the diagonal action of $\operatorname{PSL}(2, \mathbb{C})$. These quotients have been studied extensively, for example in MFK, Th. In the symplectic geometry literature these spaces are known as polygon spaces, as they parameterize $n$-sided polygons in $\mathbb{R}^{3}$ with fixed edge lengths, up to rotation [HK].

The action of $G$ on $V$ induces a symplectic action on the cotangent bundle $T^{*} V$, with moment map $\mu: T^{*} V \rightarrow \mathfrak{g}^{*}$ given by the equation

$$
\mu(p, \alpha)(x)=\alpha\left(\hat{x}_{p}\right),
$$

where $\alpha$ is a cotangent vector to $V$ at $p$, and $\hat{x}_{p}$ is the tangent vector at $p$ induced by the infinitesimal action of $x \in \mathfrak{g}$. For $\lambda \in \mathfrak{g}^{*}$, let $\phi_{\lambda}: \mu^{-1}(\lambda) \rightarrow V$ be the canonical projection to $V$. Given an equivariant ample line bundle $L$ on $V$, we define the space

$$
M_{\lambda, L}:=\mu^{-1}(\lambda) /_{\phi_{\lambda}^{*} L} G
$$


to be the GIT quotient of $\mu^{-1}(\lambda)$ by $G$. If $\lambda$ is a regular value of $\mu$, then $G$ acts locally freely on $\mu^{-1}(\lambda)$, and the quotient is geometric; in particular, it is independent of $L$. Thus when $\lambda$ is a regular value we will drop $L$ from the notation.

Proposition 1.3. If $\lambda \in \mathfrak{g}^{*}$ is a regular value of $\mu$, then $\operatorname{Im} \phi_{\lambda}=V^{\ell f}$, and the fibers of $\phi_{\lambda}$ are affine spaces of dimension $\operatorname{dim} V-\operatorname{dim} G$.

Proof. For any point $p \in V$, we have an exact sequence of $k$-vector spaces 2

$$
0 \rightarrow\{\alpha \mid \mu(p, \alpha)=0\} \rightarrow T_{p}^{*} V \stackrel{\mu(p,-)}{\longrightarrow} \mathfrak{g}^{*} \rightarrow \mathfrak{s t a} \mathfrak{b}(p)^{*} \rightarrow 0
$$

where $\mathfrak{s t a b}(p)^{*}=\mathfrak{g}^{*} / \mathfrak{s t a} \mathfrak{b}(p)^{\perp}$ is the Lie coalgebra of the stabilizer of $p$ in $G$. By exactness of (2) at $\mathfrak{g}^{*}, p$ is in the image of $\phi_{\lambda}$ if and only if $\lambda \cdot \mathfrak{s t a b}(p)=0$. If $p \in V^{\ell f}$, then $\mathfrak{s t a b}(p)=0$, thus $p \in \operatorname{Im} \phi_{\lambda}$. Conversely, suppose that $p \in \operatorname{Im} \phi_{\lambda}$. Since $\lambda$ is a regular value of $\mu, G$ acts locally freely on $\mu^{-1}(\lambda)$, and therefore $\operatorname{Stab}(p) \subseteq G$ acts locally freely on $\phi_{\lambda}^{-1}(p)$. But $\phi_{\lambda}^{-1}(p)$ is a torsor for the vector space $\{\alpha \mid \mu(p, \alpha)=$ $0\}$, and any torus action on an affine space has a fixed point. Hence $\operatorname{Stab}(p)$ must be finite, and therefore $p \in V^{\ell f}$. Finally, we see that if $p \in V^{\ell f}=\operatorname{Im} \phi_{\lambda}$, then $\operatorname{dim} \phi_{\lambda}^{-1}(p)=\operatorname{dim}\{\alpha \mid \mu(p, \alpha)=0\}=\operatorname{dim} T_{p}^{*} V-\operatorname{dim} \mathfrak{g}^{*}$.

Consider the nonseparated prevariety

$$
X^{\ell f}=V^{\ell f} / G=\bigcup_{i \in I} V^{s t}\left(L_{i}\right) / G=\bigcup_{i \in I} X_{i},
$$

where two GIT quotients $X_{i}$ and $X_{j}$ are glued together along the open set of points which are simultaneously stable for both $L_{i}$ and $L_{j}$. Proposition 1.3 has the following immediate corollary.

Corollary 1.4. For any regular value $\lambda$ of $\mu, M_{\lambda}=\mu^{-1}(\lambda) / G$ is isomorphic to the total space of an affine bundle over $X^{\ell f}$, modeled on the cotangent bundle.

Remark 1.5. The space $M_{\lambda}$ is sometimes known as the twisted cotangent bundle to the stack $V / G$. In this language, Proposition 1.3 says that the twisted cotangent bundle has support $X^{\ell f} \subseteq V / G$, and that over its support it has constant rank. This formulation generalizes to the nonabelian case if we replace $X^{\ell f}$ by the locus of points in $V / G$ whose infinitesimal stabilizer is perpendicular to $\lambda$, and interpret rank to be the difference between the dimension of the fiber and the dimension of the stabilizer group at a point. If $\lambda$ is generic, the stabilizer Lie algebra is perpendicular to $\lambda$ if and only if it is nilpotent.

\section{A cohomological conjecture}

The purpose of this section will be to consider the cohomological implications of Corollary 1.4, taken over the complex numbers, focusing on the case of toric and hypertoric varieties. Specifically, there is a natural map on cohomology

$$
\Psi: H^{*}\left(M_{\lambda} ; \mathbb{R}\right) \cong H^{*}\left(X^{\ell f} ; \mathbb{R}\right) \rightarrow \bigoplus_{i \in I} H^{*}\left(X_{i} ; \mathbb{R}\right)
$$

given by the inclusions of each $X_{i}$ into $X^{\ell f}$.

Conjecture 2.1. If $\mathcal{O}_{V}^{G} \cong \mathbb{C}$, then $\Psi$ is injective.

\footnotetext{
${ }^{2}$ The analogous exact sequence in the context of representations of quivers first appeared in $\mathrm{CB}$, and was used to count points on quiver varieties over finite fields in [CBVdB].
} 
Remark 2.2. The hypothesis $\mathcal{O}_{V}^{G} \cong \mathbb{C}$ says precisely that the GIT quotients $\left\{X_{i}\right\}$ are projective, and without this assumption Conjecture 2.1 fails. Specifically, let $G=\mathbb{C}^{\times}$act on $V=\mathbb{C}^{2}$ with eigenvalues \pm 1 . There are two GIT quotients, namely $X_{1}=(V \backslash \mathbb{C} \times\{0\}) / G \cong \mathbb{C}$ and $X_{2}=(V \backslash\{0\} \times \mathbb{C}) / G \cong \mathbb{C}$, neither of which has any nontrivial cohomology. On the other hand, $X^{\ell f}=V \backslash\{0\} / G$ is an affine line with a double point, which is weakly homotopy equivalent to the 2 -sphere. So $\Psi$ fails to be injective in degree 2 .

Theorem 2.3. Conjecture 2.1 holds for the polygon spaces of Example 1.2.

Proof. Konno studies a map from $H^{*}\left(M_{0, L} ; \mathbb{R}\right)$ to $\bigoplus H^{*}\left(X_{i} ; \mathbb{R}\right)$ for generic $L$, which he shows is injective [K2, 7.6]. He also observes that $M_{0, L}$ is diffeomorphic to $M_{\lambda}$, and while this diffeomorphism is not canonical, the induced isomorphism of cohomology rings is. It is then not hard to see that the map studied by Konno agrees with our map $\Psi$.

Remark 2.4. If Conjecture 2.1 were true, it would imply that $H^{*}\left(M_{\lambda} ; \mathbb{R}\right)$ is level, which means that every nonzero class divides a nonzero class of top degree. (One may think of this property as a generalization of Poincaré duality to the situation where the top degree cohomology need not be one dimensional.) Indeed, given a nonzero class $\alpha \in H^{*}\left(M_{\lambda} ; \mathbb{R}\right)$, Conjecture 2.1 would tell us that $\alpha$ restricts to a nonzero class $\alpha_{i} \in H^{*}\left(X_{i} ; \mathbb{R}\right)$ for some $i$. Then, by Poincaré duality for $X_{i}$, there exists a class $\beta_{i} \in H^{*}\left(X_{i} ; \mathbb{R}\right)$ such that $\alpha_{i} \cdot \beta_{i}$ is a nonzero class on $X_{i}$ of top degree. Now it suffices to show that $\beta_{i}$ is the restriction of a class in $H^{*}\left(M_{\lambda} ; \mathbb{R}\right) \cong H^{*}\left(X^{\ell f} ; \mathbb{R}\right)$. But this follows from Kirwan surjectivity [Ki, 5.4], which tells us that the map $H_{G}^{*}(V ; \mathbb{R}) \rightarrow H^{*}\left(X_{i} ; \mathbb{R}\right)$ is surjective. This map factors through $H_{G}^{*}\left(V^{\ell f} ; \mathbb{R}\right) \cong H^{*}\left(X^{\ell f} ; \mathbb{R}\right)$, which implies that the map from $H^{*}\left(X^{\ell f} ; \mathbb{R}\right)$ to $H^{*}\left(X_{i} ; \mathbb{R}\right)$ is surjective as well.

We note that Conjecture 2.1 is in fact equivalent to the statement that $H^{*}\left(M_{\lambda} ; \mathbb{R}\right)$ is level and the fundamental cycles of the GIT quotients $\left\{X_{i}\right\}$ generate the top homology of $X^{\ell f}$. This is essentially the strategy used by Konno to prove Theorem 2.3 .

The rest of Section 2 will be devoted to understanding and proving Conjecture 2.1 in the toric case (Theorem 2.8). Let $V=\mathbb{C}^{n}, T^{n}$ the coordinate torus acting on $V$, and $G$ is a codimension $d$ subtorus of $T^{n}$. Let $\chi=\left(\chi_{1}, \ldots, \chi_{n}\right)$ be an $n$-tuple of integers, which we interpret as a multiplicative character $T^{n} \rightarrow \mathbb{C}^{*}$ by the equation

$$
\chi(t)=t_{1}^{\chi_{1}} \ldots t_{n}^{\chi_{n}} .
$$

Let $L_{\chi}$ be the $G$-equivariant line bundle on $V$ obtained from twisting the trivial bundle by the restriction of $\chi$ to $G$. The GIT quotients $V / L_{L_{\chi}} G$ are called toric varietie 3 , and the symplectic quotients $M_{\lambda, L_{\chi}}$ are called hypertoric varieties. Both are intricately related to various combinatorial data associated to the subtorus $G \subseteq T^{n}$ and the character $\chi$, as we describe below.

Let $T^{d}=T^{n} / G$ with Lie algebra $\mathfrak{t}^{d}=\mathfrak{t}^{n} / \mathfrak{g}$. This algebra is equipped with an integer lattice (the kernel of the exponential map), and therefore with a canonical real form $\mathfrak{t}_{\mathbb{R}}^{d} \subseteq \mathfrak{t}^{d}$. Let $a_{1}, \ldots, a_{n} \in \mathfrak{t}_{\mathbb{R}}^{d}$ be the projections of the standard basis

\footnotetext{
${ }^{3} \mathrm{An}$ introduction to toric varieties from the GIT perspective can be found in $\mathrm{Pr}$.
} 
vectors in $\mathfrak{t}^{n}$. For all $i \in\{1, \ldots, n\}$, we define half spaces

(4)

$F_{i}^{\chi}:=\left\{x \in\left(\mathfrak{t}_{\mathbb{R}}^{d}\right)^{*} \mid x \cdot a_{i}+\chi_{i} \geq 0\right\} \quad$ and $\quad G_{i}^{\chi}:=\left\{x \in\left(\mathfrak{t}_{\mathbb{R}}^{d}\right)^{*} \mid x \cdot a_{i}+\chi_{i} \leq 0\right\}$.

The geometry of the toric variety $V /{ }_{L_{\chi}} G$ is completely controlled by the polyhedron

$$
\Delta^{\chi}:=\bigcap_{i=1}^{n} F_{i}^{\chi}
$$

(see $[\mathrm{Pr}]$ and the references therein). The line bundle $L_{\chi}$ is nice if and only if $\Delta^{\chi}$ is a simple polyhedron of dimension $d$, which means that exactly $d$ facets meet at each vertex. In this case, we have the following well-known theorem (see, for example, HS, 2.11]).

Theorem 2.5. If $L_{\chi}$ is nice, then the $T^{d}$-equivariant cohomology ring of $V / L_{L_{\chi}} G$ is isomorphic to the Stanley-Reisner ring of the normal fan to $\Delta^{x}$.

In particular, this implies that the Betti numbers of toric varieties are given by combinatorial invariants of polytopes $[\mathrm{St}$. Theorem 2.5 has the following analogue for hypertoric varieties, which appeared in this form in [HS], and in a different but equivalent form in [K1.

Theorem 2.6. If $\lambda$ is generic, then the $T^{d}$-equivariant cohomology ring of $M_{\lambda}$ is isomorphic to the Stanley-Reisner ring of the matroid associated to the vector collection $\left\{a_{1}, \ldots, a_{n}\right\}$.

A consequence of this fact is that the Betti numbers of hypertoric varieties are combinatorial invariants of matroids [HS, 6.6]. It is also possible to obtain this result by counting points on $M_{\lambda}$ over finite fields, as in [PW] or [Ha].

Remark 2.7. The set of equivalence classes of nice line bundles on $V$ corresponds to the set of possible combinatorial types of $\Delta^{\chi}$, which in turn is indexed by triangulations of the oriented matroid determined by the vectors $\left\{a_{1}, \ldots, a_{n}\right\}$ [Sa].

Theorem 2.8. If $\mathcal{O}_{V}^{G} \cong \mathbb{C}$, then the map $\Psi$ of Equation (3) is injective.

Remark 2.9. Using Theorems 2.5] and 2.6, it is relatively easy to prove injectivity of the natural lift of $\Psi$ to a map in $T^{d}$-equivariant cohomology. Theorem 2.8, however, does not follow formally from this fact. For our proof it is necessary to use different descriptions of the cohomology rings of toric and hypertoric varieties (see Theorems 2.11 and 2.12).

Remark 2.10. Since we know that the cohomology ring of a hypertoric variety is level [HS, §7], Remark 2.4 tells us that Theorem 2.8 is equivalent to the statement that the fundamental cycles of the projective toric varieties $\left\{X_{i}\right\}$ generate $H^{2 d}\left(X^{\ell f}\right)$. This is a little bit surprising, as $X^{\ell f}$ will often contain proper but nonprojective toric varieties as open subsets. Theorem 2.8 asserts that the fundamental cycle of any such subvariety can be expressed as a linear combination of the fundamental cycles of the $\left\{X_{i}\right\}$. Our proof, though not in this language, will roughly follow this line of attack. 
Proof of Theorem 2.8, We begin by noting that the definitions given in Equations (41) and (51) make sense for any vector $\chi=\left(\chi_{1}, \ldots, \chi_{n}\right) \in \mathbb{R}^{n} \cong\left(\mathfrak{t}_{\mathbb{R}}^{n}\right)^{*}$, regardless of whether the coordinates of $\chi$ are integers. For any subset $A \subseteq\{1, \ldots, n\}$, let

$$
\Delta_{A}^{\chi}:=\bigcap_{i \in A^{c}} F_{i}^{\chi} \cap \bigcap_{j \in A} G_{j}^{\chi},
$$

and note that $\Delta_{\emptyset}^{\chi}=\Delta^{\chi}$. In general, one should imagine $\Delta_{A}^{\chi}$ as the polytope obtained from $\Delta^{\chi}$ by "flipping" it over the hyperplane $F_{i}^{\chi} \cap G_{i}^{\chi}$ for each $i \in A$. We will call $\chi$ simple if for every $A \subseteq\{1, \ldots, n\}, \Delta_{A}^{\chi}$ is either empty or simple of dimension $d$. In particular, if $\chi$ is an integer vector and $\chi$ is simple, then $L_{\chi}$ is nice. If $\Delta_{A}^{\chi}$ is nonempty, then it is bounded if and only if the vectors $\left\{\varepsilon_{1}(A) a_{1}, \ldots, \varepsilon_{n}(A) a_{n}\right\}$ span $\mathfrak{t}_{\mathbb{R}}^{d}$ over the nonnegative real numbers, where $\varepsilon_{i}(A)=(-1)^{|A \cap\{i\}|}$. We call such an $A$ admissible. Note that the assumption $\mathcal{O}_{V}^{G}=\mathbb{C}$ is equivalent to the assumption that the empty set is admissible.

The volume function $\operatorname{Vol} \Delta_{A}^{\chi}$ is locally polynomial in $\chi$. More precisely, for every simple $\chi \in\left(\mathfrak{t}_{\mathbb{R}}^{n}\right)^{*}$ and every admissible $A \subseteq\{1, \ldots, n\}$, there exists a polynomial $P_{A}^{\chi} \in \operatorname{Sym}^{d} \mathfrak{t}_{\mathbb{R}}^{n}$ such that for every simple $\eta \in\left(\mathfrak{t}_{\mathbb{R}}^{n}\right)^{*}$ sufficiently close to $\chi$, we have

$$
\operatorname{Vol} \Delta_{A}^{\eta}=P_{A}^{\chi}(\eta)
$$

We will refer to $P_{A}^{\chi}$ as the volume polynomial of $\Delta_{A}^{\chi}$, and write $P^{\chi}=P_{\emptyset}^{\chi}$. The cohomology rings of toric and hypertoric varieties may be described in terms of these volume polynomials as follows. Let $\left\{x_{1}, \ldots, x_{n}\right\}$ be the coordinate basis for $\left(\mathfrak{t}_{\mathbb{R}}^{n}\right)^{*}$, and $\left\{\partial_{1}, \ldots, \partial_{n}\right\}$ the dual basis for $\mathfrak{t}_{\mathbb{R}}^{n}$. There is a natural action of Sym $\mathfrak{t}_{\mathbb{R}}^{n}$ on $\operatorname{Sym}\left(\mathfrak{t}_{\mathbb{R}}^{n}\right)^{*}$ given by differentiation of polynomials, and this restricts to an action of the subring Sym $\mathfrak{g}_{\mathbb{R}}$.

Theorem 2.11 ([GS, $\overline{\mathrm{KP}})$. If $\chi \in\left(\mathfrak{t}_{\mathbb{R}}^{n}\right)^{*}$ is simple with integer coordinates, then

$$
H^{*}\left(V / L_{L_{\chi}} G ; \mathbb{R}\right) \cong \operatorname{Sym} \mathfrak{g}_{\mathbb{R}} / \operatorname{Ann}\left(P^{\chi}\right)
$$

where $\operatorname{Ann}\left(P^{\chi}\right)=\left\{\partial \in \operatorname{Sym} \mathfrak{g}_{\mathbb{R}} \mid \partial \cdot P^{\chi}=0\right\}$.

Theorem $2.12([\underline{\mathrm{HS}}, 7.1])$. If $\chi \in\left(\mathfrak{t}_{\mathbb{R}}^{n}\right)^{*}$ is simple with integer coordinates, then for any $\lambda \in \mathfrak{t}^{d}$,

$$
H^{*}\left(M_{\lambda, L_{\chi}} ; \mathbb{R}\right) \cong \operatorname{Sym} \mathfrak{g}_{\mathbb{R}} / \operatorname{Ann}\left\{P_{A}^{\chi} \mid A \text { admissible }\right\} .
$$

If we fix $\lambda$ to be a regular value of $\mu$, then we have already observed that the left-hand side of the isomorphism of Theorem 2.12 does not depend on $\chi$. From this it follows that the right-hand side does not depend on $\chi$ either; in other words, the linear span

$$
U=\mathbb{R}\left\{P_{A}^{\chi} \mid A \text { admissible }\right\}
$$

is independent of $\chi$. Since the empty set is admissible, $P^{\chi}$ is contained in $U$ for all simple integral $\chi$, and this inclusion induces the canonical map from $H^{*}\left(M_{\lambda} ; \mathbb{R}\right)$ to $H^{*}\left(V / L_{L_{\chi}} G ; \mathbb{R}\right)$. The kernel of $\Phi$ is therefore equal to the image in $H^{*}\left(M_{\lambda}\right)$ of the set of polynomials in $\operatorname{Sym}_{\mathbb{R}}^{d}$ that annihilate $P^{\chi}$ for every simple integral $\chi$. To prove Theorem 2.8 we need to show that every such polynomial annihilates $U$; in other words, we must show that every polynomial of the form $P_{A}^{\chi}$ for some simple $\chi$ and admissible $A$ can be expressed as a linear combination of polynomials of the form $P^{\chi}$, where $\chi$ is allowed to vary. 
To this end, let $\mathcal{F}$ be the infinite dimensional vector space consisting of all realvalued functions on $\left(\mathfrak{t}_{\mathbb{R}}^{d}\right)^{*}$ modulo those which are zero on a dense open set, and let $\mathcal{F}^{b d}$ be the subspace spanned by functions with bounded support. For all subsets $A \subseteq\{1, \ldots, n\}$, let

$$
W_{A}=\mathbb{Q}\left\{\mathbf{1}_{\Delta_{A}^{\chi}} \mid \chi \text { simple and integral }\right\}
$$

be the subspace of $\mathcal{F}$ spanned by the characteristic functions of polyhedra $\Delta_{A}^{\chi}$ for simple and integral $\chi$, and let

$$
W_{A}^{b d}=W_{A} \cap \mathcal{F}^{b d} .
$$

Note that $W_{A}^{b d}=W_{A}$ if and only if $A$ is admissible.

Lemma 2.13. For all $A, A^{\prime} \subseteq\{1, \ldots, n\}, W_{A}^{b d}=W_{A^{\prime}}^{b d}$.

Proof. We may immediately reduce to the case where $A^{\prime}=A \cup\{k\}$ for some $k$. Fix a simple $\chi \in\left(\mathfrak{t}_{\mathbb{R}}^{n}\right)^{*}$. Let $\tilde{\chi} \in\left(\mathfrak{t}^{n}\right)^{*}$ be another simple element obtained from $\chi$ by putting $\tilde{\chi}_{i}=\chi_{i}$ for all $i \neq k$, and $\tilde{\chi}_{k}=N$ for some integer $N \gg 0$. Then $\Delta_{A}^{\chi} \subseteq \Delta_{A}^{\tilde{\chi}}$, and

$$
\begin{aligned}
\Delta_{A}^{\tilde{\chi}} \backslash \Delta_{A}^{\chi} & =\bigcap_{i \in\left(A^{\prime}\right)^{c}} F_{i}^{\chi} \cap \bigcap_{j \in A} G_{j}^{\chi} \cap\left(F_{k}^{\tilde{\chi}} \backslash F_{k}^{\chi}\right) \\
& =\Delta_{A^{\prime}}^{\chi} \cap F_{k}^{\tilde{\chi}} \backslash F_{k}^{\chi} \cap G_{k}^{\chi} .
\end{aligned}
$$

Since the complement of the hyperplane $F_{k}^{\chi} \cap G_{k}^{\chi}$ is dense and open in $\left(\mathfrak{t}_{\mathbb{R}}^{d}\right)^{*}$, this computation yields the equality

$$
\mathbf{1}_{\Delta_{A}^{\tilde{\chi}}}-\mathbf{1}_{\Delta_{A}^{\chi}}=\mathbf{1}_{\Delta_{A^{\prime}}^{\chi}} \cdot \mathbf{1}_{F_{k}^{\tilde{\chi}}}
$$

in $\mathcal{F}$. Suppose that $f \in \mathcal{F}^{b d}$ can be written as a linear combination of functions of the form $\mathbf{1}_{\Delta_{A^{\prime}}^{\chi}}$. Choosing $N$ large enough that the support of $f$ is contained in the half space $F_{k}^{\tilde{\chi}}$, it follows that $f$ can be written as a linear combination of functions of the form $\mathbf{1}_{\Delta_{A}^{\chi}}$, hence $W_{A^{\prime}}^{b d} \subseteq W_{A}^{b d}$. The reverse inclusion is obtained by an identical argument.

By Lemma 2.13, we may write

$$
\mathbf{1}_{\Delta_{A}^{\chi}}=\sum_{j=1}^{m} \alpha_{j} \mathbf{1}_{\Delta^{\eta^{j}}}
$$

for any simple $\chi$ and admissible $A$, where $\alpha_{j} \in \mathbb{Q}$ and $\eta^{j}$ is a simple integral element of $\left(\mathfrak{t}^{n}\right)^{*}$ for all $j \leq m$. Taking volumes of both sides of the equation, we have

$$
P_{A}^{\chi}(\chi)=\sum_{j=1}^{m} \alpha_{j} P^{\eta^{j}}\left(\eta^{j}\right) .
$$

Furthermore, we can assume (by the proof of Lemma 2.13) that for all $j \leq m$ and all $i \leq n$, the $i^{\text {th }}$ coordinate $\eta_{i}^{j}$ of $\eta^{j}$ is either equal to $\chi_{i}$, or to some large number $N \gg 0$. Equation (7) still holds if we wiggle these large numbers a little bit, hence the polynomial on the right-hand side must be independent of the variable 

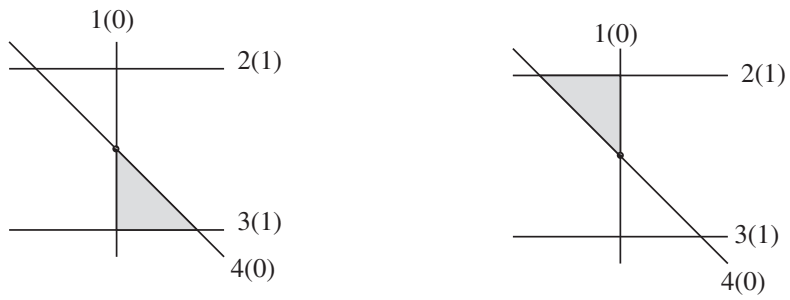

FiguRe 1. The number outside of the parentheses denotes the index $i$ of the half space, and the number inside denotes the value of $\chi_{i}$. This value is equal (up to sign) to the distance from the boundary of the $i^{\text {th }}$ half space to the origin of $\left(\mathfrak{t}_{\mathbb{R}}^{d}\right)^{*}$, which is marked with a black dot.

$\eta_{i}^{j}$ whenever $\eta_{i}^{j} \neq \chi_{i}$. Thus we may substitute $\chi$ for each $\eta^{j}$, and we obtain the equation

$$
P_{A}^{\chi}(\chi)=\sum_{j=1}^{m} \alpha_{j} P^{\eta^{j}}(\chi)
$$

This equation clearly holds in a neighborhood of $\chi$, hence we obtain an equation of polynomials

$$
P_{A}^{\chi}=\sum_{j=1}^{m} \alpha_{j} P^{\eta^{j}} .
$$

This completes the proof of Theorem 2.8

Example 2.14. Let us consider an example in which $n=4$ and $d=2$. The picture on the left-hand side of Figure 1 shows a polytope $\Delta^{\chi}$ where $\chi=(0,1,1,0)$, and the picture on the right shows $\Delta_{\{1,4\}}^{\chi}$.

The key to the proof of Theorem 2.8 is our ability to express the characteristic function of $\Delta_{\{1,4\}}^{\chi}$ in terms of the characteristic functions of $\Delta^{\eta^{j}}$ for some finite set $\left\{\eta^{1}, \ldots, \eta^{m}\right\}$ of simple integral vectors, as we did in Equation 6 . Since $\{1,4\}$ has two elements, the procedure described in Lemma 2.13 must be iterated twice, and the result will have a total of $2^{2}=4$ terms, as illustrated in Figure 2, The first iteration exhibits $\mathbf{1}_{\Delta_{\{1,4\}}}$ as an element of $W_{\{4\}}^{b d}$ by expressing it as the difference of the characteristic functions of two (unbounded) regions. With the second iteration, we attempt to express each of these two characteristic functions as elements of $W_{\{1,4\}}^{b d}=W_{\{1,4\}}$. This attempt must fail, because each of the two functions that we try to express has unbounded support. But the failures cancel out, and we succeed in expressing the difference as an element of $W_{\{1,4\}}$.

Let's see what happens when we take volume polynomials in the equation of Figure 2. The two polytopes on the top line have different volumes, but the same volume polynomial, hence these two terms cancel. We are left with the equation

which translates into

$$
P_{\{1,4\}}^{(0,1,1,0)}=P^{(0,1,1,0)}-P^{(N, 1,1,0)},
$$

$$
\frac{1}{2}\left(-\chi_{1}+\chi_{2}-\chi_{4}\right)^{2}=\frac{1}{2}\left(\chi_{1}+\chi_{3}+\chi_{4}\right)^{2}-\left(\chi_{2}+\chi_{3}\right)\left(\chi_{1}+\chi_{4}+\frac{1}{2} \chi_{3}-\frac{1}{2} \chi_{2}\right) .
$$



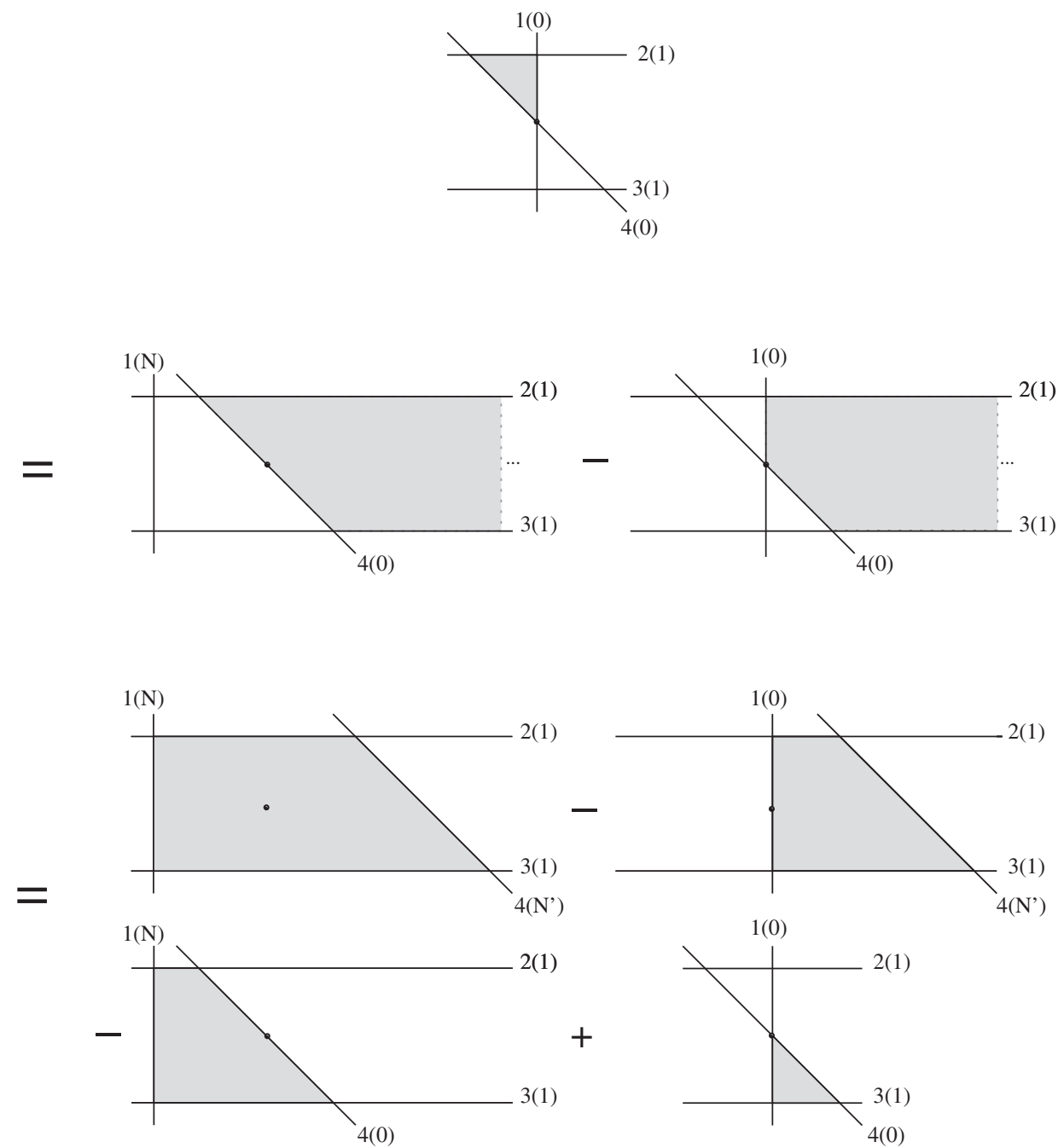

Figure 2. An equation of characteristic functions. The procedure of Lemma 2.13 has produced two undetermined large numbers, which we call $N$ and $N^{\prime}$.

\section{ACKNOWLEDGMENTS}

This paper benefited greatly from conversations with Matthias Beck and Rebecca Goldin, and from the work of William Crawley-Boevey. The author would also like to thank the referee for his valuable comments.

\section{REFERENCES}

[BD] R. Bielawski and A. Dancer. The geometry and topology of toric hyperkähler manifolds. Comm. Anal. Geom. 8 (2000), 727-760. MR.1792372 (2002c:53078)

[CB] W. Crawley-Boevey. Geometry of the moment map for representations of quivers. Compositio Math. 126 (2001), no. 3, 257-293. MR1834739 (2002g:16021) 
[CBVdB] W. Crawley-Boevey and M. Van den Bergh. Absolutely indecomposable representations and Kac-Moody Lie algebras. With an appendix by Hiraku Nakajima. Invent. Math. 155 (2004), no. 3, 537-559. MR2038196 (2004m:17032)

[DH] I. Dolgachev and Y. Hu. Variation of geometric invariant theory quotients. With an appendix by Nicolas Ressayre. Inst. Hautes Études Sci. Publ. Math. No. 87, (1998), 5-56. MR1659282 (2000b:14060)

[GS] V. Guillemin and S. Sternberg. The coefficients of the Duistermaat-Heckman polynomial and the cohomology ring of reduced spaces. Geometry, topology, $\&$ physics, 202-213, Conf. Proc. Lecture Notes Geom. Topology, IV, Internat. Press, Cambridge, MA, 1995. MR.1358618 (96k:58082)

[HP] M. Harada and N. Proudfoot. Hyperpolygon spaces and their cores. Trans. A.M.S. 357 (2005), 1445-1467. MR2115372 (2006f:53064)

[Ha] T. Hausel. Betti numbers of holomorphic symplectic quotients via arithmetic Fourier transform. Proc. of the Nat. Acad. Sci. 103 (2006), no. 16, 6120-6124 (electronic). MR 2221039 (2007m:53109)

[HS] T. Hausel and B. Sturmfels. Toric hyperkähler varieties. Doc. Math. 7 (2002), 495-534 (electronic). MR2015052 (2004i:53054)

[HK] J.C. Hausmann and A. Knutson. Polygon spaces and Grassmannians. L'Enseignement Mathematique 43 (1997), no. 1-2, 173-198. MR1460127 (98e:58035)

[Ki] F. Kirwan. Cohomology of quotients in symplectic and algebraic geometry. Mathematical Notes 31, Princeton University Press, 1984. MR766741 (86i:58050)

[KP] A. Khovanskii and A. Pukhlikov. The Riemann-Roch theorem for integrals and sums of quasipolynomials on virtual polytopes. St. Petersburg Math. J. 4 (1993), 789-812. MR.1190788 (94c:14044)

[K1] H. Konno. Cohomology rings of toric hyperkähler manifolds. Int. J. of Math. 11 (2000), no. 8, 1001-1026. MR.1797675 (2001k:53089)

[K2] H. Konno. On the cohomology ring of the HyperKähler analogue of the Polygon Spaces. Integrable systems, topology, and physics (Tokyo, 2000), 129-149, Contemp. Math., 309, Amer. Math. Soc., Providence, RI, 2002. MR.1953356 (2003k:53111)

[MFK] D. Mumford, J. Fogarty, and F. Kirwan. Geometric Invariant Theory. Third edition. Ergebnisse der Mathematik und ihrer Grenzgebiete (2) [Results in Mathematics and Related Areas (2)], 34. Springer-Verlag, 1994. MR.1304906 (95m:14012)

[N1] H. Nakajima. Instantons on ALE spaces, quiver varieties, and Kac-Moody algebras. Duke Math. J. 76 (1994), no. 2, 365-416. MR1302318 (95i:53051)

[N2] H. Nakajima. Quiver varieties and Kac-Moody algebras. Duke Math. J. 91 (1998), no. 3, 515-560. MR1604167 (99b:17033)

[N3] H. Nakajima. Quiver varieties and finite-dimensional representations of quantum affine algebras. J. Amer. Math. Soc. 14 (2001), no. 1, 145-238. MR1808477 (2002i:17023)

[Pr] N. Proudfoot. Geometric invariant theory and projective toric varieties. Snowbird lectures in algebraic geometry, 161-167, Contemp. Math. 388, Amer. Math. Soc., Providence, RI, 2005. MR2182896(2006j:14065)

[PW] N. Proudfoot and B. Webster. Intersection cohomology of hypertoric varieties. $\mathrm{J} . \mathrm{Al}-$ gebraic Geom. 16 (2007), no. 1, 39-63. MR2257319(2008m:14037)

[Sa] F. Santos. Triangulations of oriented matroids. Mem. Amer. Math. Soc. 156 (2002), no. 741. MR 1880595 (2003b:52013)

[St] R. Stanley. The number of faces of a simplicial convex polytope. Adv. in Math. 35 (1980), no. 3, 236-238. MR.563925 (81f:52014)

[Th] M. Thaddeus. Geometric invariant theory and flips. J. Amer. Math. Soc. 9 (1996), no. 3, 691-723. MR1333296 (96m:14017)

Department of Mathematics, University of Oregon, Eugene, Oregon 97403 